This item was submitted to Loughborough's Research Repository by the author.

Items in Figshare are protected by copyright, with all rights reserved, unless otherwise indicated.

\title{
Impact of data aggregation approaches on the relationships between operating speed and traffic safety
}

PLEASE CITE THE PUBLISHED VERSION

https://doi.org/10.1016/j.aap.2018.06.007

\section{PUBLISHER}

(C) Elsevier

\section{VERSION}

AM (Accepted Manuscript)

\section{PUBLISHER STATEMENT}

This paper was accepted for publication in the journal Accident Analysis and Prevention and the definitive published version is available at https://doi.org/10.1016/j.aap.2018.06.007.

\section{LICENCE}

CC BY-NC-ND 4.0

\section{REPOSITORY RECORD}

Yu, Rongjie, Mohammed Quddus, Xuesong Wang, and Kui Yang. 2019. "Impact of Data Aggregation Approaches on the Relationships Between Operating Speed and Traffic Safety". figshare. https://hdl.handle.net/2134/36521. 

between operating speed and traffic safety

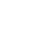

\author{
Rongjie Yu*, Ph.D. \\ School of Transportation Engineering, Tongji University \\ 4800 Cao'an Road, 201804, Shanghai, China
}

Key Laboratory of Road and Traffic Engineering of Ministry of Education 4800 Cao'an Road, 201804, Shanghai, China

*Corresponding Author: yurongjie@,tongji.edu.cn

\title{
Mohammed Quddus, Ph.D.
}

School of Architecture, Building and Civil Engineering, Loughborough University, Loughborough LE11 3TU, United Kingdom

\author{
Xuesong Wang, Ph.D. \\ School of Transportation Engineering, Tongji University \\ 4800 Cao'an Road, 201804, Shanghai, China
}

Key Laboratory of Road and Traffic Engineering of Ministry of Education 4800 Cao'an Road, 201804, Shanghai, China

\author{
Kui Yang, Ph.D. Student \\ School of Transportation Engineering, Tongji University \\ 4800 Cao'an Road, 201804, Shanghai, China \\ Key Laboratory of Road and Traffic Engineering of Ministry of Education \\ 4800 Cao'an Road, 201804, Shanghai, China \\ ("corresponding author) \\ June 2018
}




\begin{abstract}
The impact of operating speed on traffic crash occurrence has been a controversial topic in the traffic safety discipline as some studies reported a positive association whereas others indicated a negative relationship between speed and crashes. Two major issues thought to be accountable for such conflicting findings are the application of inappropriate statistical methods and the use of sample datasets with varying levels of aggregation. The main objective of this study is therefore to investigate the impacts of data aggregation schemes on the relationships between operating speed and traffic safety. A total of three aggregation approaches were examined: (1) a segment-based dataset in which crashes are grouped by roadway segment, (2) a scenario-based dataset where crashes are aggregated by traffic operating scenarios, and (3) a disaggregated crash-level dataset consisting of information from individual crashes. The first two aggregation approaches were used in examining the relationships between operating speed and crash frequency using Bayesian random-effects negative binomial models. The third disaggregated crash risk analysis was conducted utilizing Bayesian random-effects logistic regression models. From the modelling results, it has been concluded that the scenario-based approach shared similar findings with those of the disaggregated crash risk analysis approach in which a U-shaped relationship between operating speed and crash occurrence was identified. However, the commonly adopted segment-based aggregation approach revealed a monotonous negative relationship between speed and crash frequency. The implications of the different analyses results and the potential applications of the results on speed management systems have therefore been discussed.
\end{abstract}

Keywords: Speed and crashes relationship, Bayesian random-effects model, Urban expressway traffic safety, Crash aggregation approach.

\title{
Introduction
}

Speed management interventions are introduced to smooth traffic flow and enhance roadway capacity and safety. Such interventions primarily include fundamental speed limit settings (e.g. Fitzpatrick et al., 2016), Variable Speed Limits (VSL) in the Active Traffic Management Systems (e.g. Mirshahi et al., 2007) and safety improvement countermeasures such as traffic calming measures (e.g. Moreno and Garcia, 2013). However, both speed limit settings and countermeasure selections heavily rely on the in-depth understandings of the quantitative relationships between operating speeds and traffic safety. More specifically, studies were conducted to identify at which operating speed there is a high probability for crash occurrence and then countermeasures were further designed to alleviate or eliminate these conditions.

Given the importance of analyzing the relationships between operating speed and 
1 traffic safety, a few studies have established statistical models between operating

2 speed and crash occurrence. However, since traffic crashes are random and sporadic 3 events with low occurrence probabilities (AASHTO, 2010), spatio-temporal 4 aggregations are needed when formulating the analysis datasets. During the 5 aggregation, raw speed information captured by the traffic sensing detectors were also 6 assembled; operating speed data prior to crash occurrence were mixed with operating 7 speed data under normal conditions.

9 For instance, the widely adopted safety performance functions (SPFs) were developed 10 using crash frequency by segment as the dependent variable (Abdel-Aty and Radwan, 11 2000); where raw speed data were processed to work out average speed for each 12 segment over a certain period of time as an independent variable. Therefore, the 13 identified relationships were basically an association between segment-level crash 14 frequency and average operating speed in which the features of operating speeds prior 15 to crash occurrence could not be analyzed.

17 Given the crash aggregation limitations, different analysis approaches have been 18 utilized in order to unveil the effects of operating speed characteristics on crash 19 occurrence. Table 1 has summarized a few studies with similar research objectives; 20 comparisons were conducted from the aspects of crash data aggregation level, the 21 nature of assembled speed information in the analyses datasets, and their primary 22 findings. 
Table 1 Literature that analyzed relationships between speed and crash occurrence

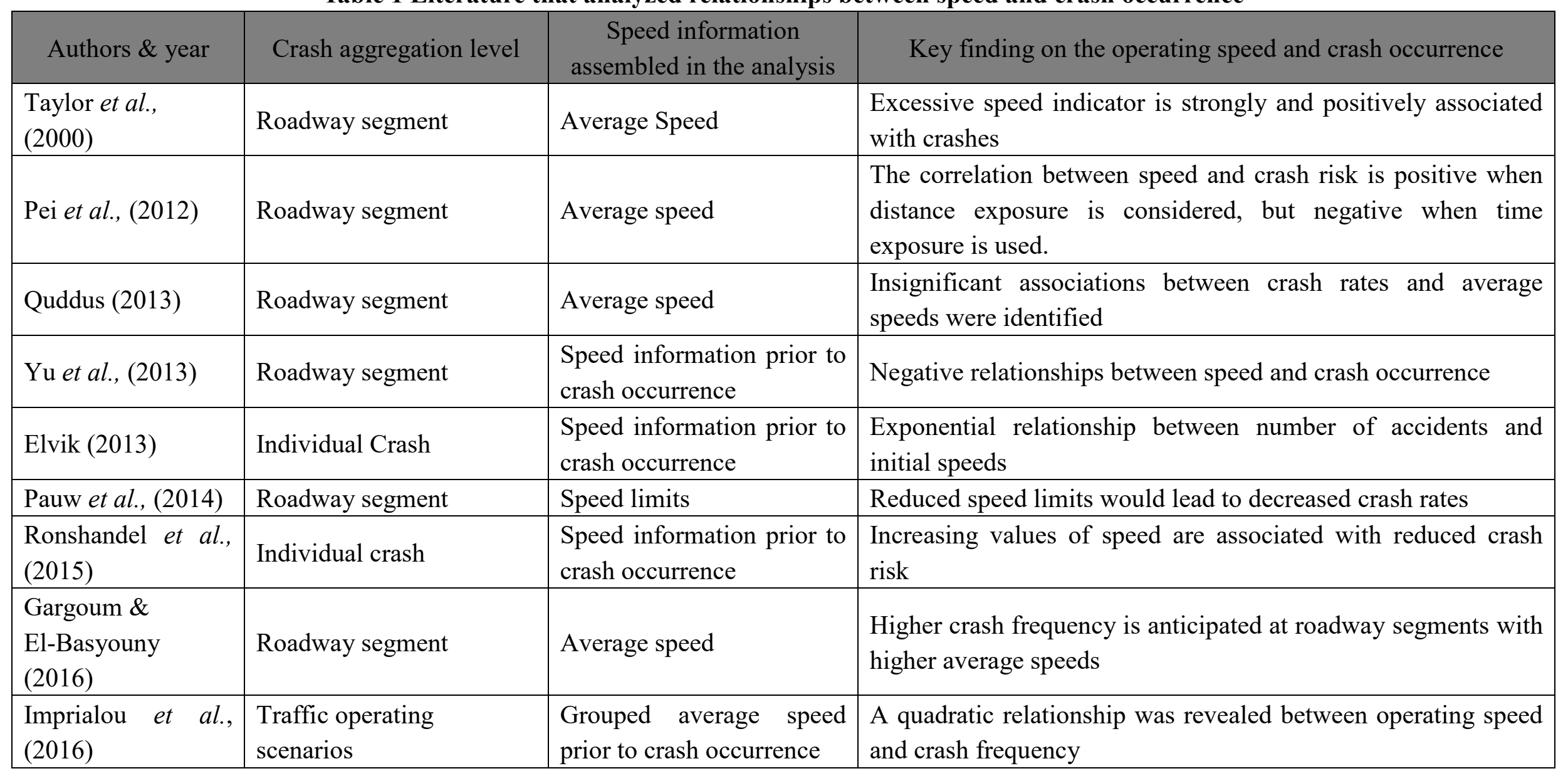


From Table 1 it can be seen that previous studies utilized crash aggregation at three levels: (1) segment-based, (2) scenario-based, and (3) individual-crash based. For the segment and scenario-based studies, crash frequency (or crash counts) was used as a dependent variable; while for the individual-crash based approach, the dichotomous crash and non-crash outcome was employed. Instead of using the average speed, several studies (Elvik, 2013; Yu et al., 2013; Imprialou et al., 2016) have tried to employ the operating speeds just prior to crash occurrence. However, the analyses conducted by using data at different levels of crash aggregation led to inconsistent results as shown in Table 1.

There is a dearth of research in investigating the reasons for conflicting findings and identifying the optimal way of integrating crash and speed data. Therefore, the purpose of this research is to identify the impacts of crash data aggregation approaches on the relationships between operating speeds and traffic safety. More specifically, the abovementioned three crash aggregation levels were compared by using speed data prior to crash occurrence.

Data from Shanghai urban expressway systems were utilized here. Firstly, the segment-based and scenario-based approaches were compared with Bayesian random-effects negative binomial models. Then, disaggregate crash risk analyses were conducted for four subgroups of crashes separately using Bayesian random-effects logistic regression modeling technique, where crashes were classified by operating speeds prior to crash occurrence. Finally, the relationships between operating speed and traffic safety were concluded. In addition, the advantages and disadvantages of the adopted aggregation approaches were discussed along with the implications of their applications on safety improvement and management.

\section{Data Preparation}

Shanghai urban expressway system was selected as the study area due to the following two reasons: (1) Shanghai urban expressway systems have relatively high-dense inductive loop detectors as a traffic sensing system with an average spacing distance of 650 meters (compared to an average of around 800 meters found in most studies (e.g. Xu et al., 2013; Abdel-Aty et al., 2007), which could provide high quality traffic flow data for the analyses; (2) traffic crashes occurred on the urban expressway system hold accurate crash locations and occurrence time since the crash records were checked with the full-coverage video surveillance system. Therefore, speed data prior to crash occurrence could be obtained accurately.

A total of three datasets were utilized: (1) crash data of September, 2013; (2) roadway geometric characteristics; and (3) traffic data by road segment collected by loop detectors aggregated at 2-minute interval. Crashes occurred on Shanghai urban 
expressways were recorded by using a stake number as reference for their location description, where stake numbers are non-repetitive marked along the roadway network. Based on the stake numbers, upstream and downstream loop detectors corresponding to crashes could be matched. In addition, considering the geometric and traffic flow features of the expressway network, roadway segments in both directions were treated as independent to each other in this study.

In order to identify the impacts of crash data aggregations on the relationships between operating speeds and traffic safety, three different levels of data aggregation were formulated: two for analyzing crash frequency and the other is to examine individual crash risk. The datasets are briefly discussed below.

\section{Datasets for the crash frequency analyses}

14 The pre-crash traffic conditions data were then aggregated with two different approaches for the crash frequency analyses: (1) segment-based approach and (2) scenario-based approach. The pre-crash traffic conditions were represented by a 6-minute interval operating condition (average operating speed and traffic volume) prior to each crash occurrence; the 2-minute raw traffic condition data were aggregated into 6-minute intervals with the purpose of reducing data collection noises, which was also adopted by Ahmed and Abdel-Aty (2012).

For the segment-based approach, crashes were aggregated based on roadway segments. The Shanghai urban expressway system was split into 206 roadway segments using on-ramps and off-ramps as dividing points. For the roadway segments, there are 4 different types of ramp combinations (see Figure 1 for illustration). It was envisaged that a segment with on-ramp and on-ramp (Ramp type 1) may be different from a segment with on-ramp and off-ramp (Ramp type 2) due to the converging and diverging traffic operation characteristics. Therefore, this categorical variable was used in the segment-based analysis. Through the aggregation process, each roadway segment may result in zero crash, one crash, or multiple crashes; the operating speed and traffic volume information variables were then calculated using the following algorithm: (1) if no crash was occurred on a segment within the study period, average operating speed and traffic volume (from 6-minute intervals) for the segment were used; (2) if only one crash was reported on a segment, the corresponding pre-crash traffic status was then utilized; (3) if multiple crashes were happened on a segment, averaged pre-crash traffic conditions corresponding to these crashes were applied. In addition to these traffic variables, geometric characteristics of the roadway 38 segments were obtained from online street-view map (Data@NavInfo) since no 39 detailed design files were available; and the summary statistics of the segment based 40 dataset are presented in Table 2. 


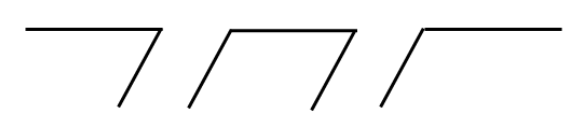

Ramp type 1

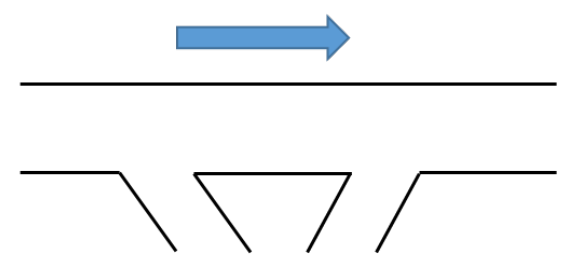

Ramp type 3

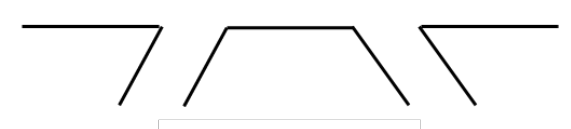

Ramp type 2

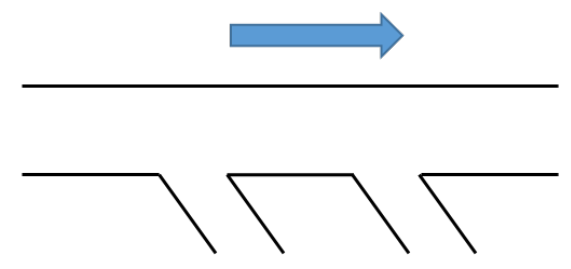

Ramp type 4
2

\begin{tabular}{|c|c|c|}
\hline Variable & Description & Summary Statistics \\
\hline Length & Roadway segment length & $\begin{array}{l}\text { Mean: } 944.5(\mathrm{~m}) \\
\text { Standard Deviation: } 585.8(\mathrm{~m})\end{array}$ \\
\hline $\begin{array}{l}\text { Lane } \\
\text { Number }\end{array}$ & Number of lanes & $\begin{array}{l}2 \text { lane: } 59 \text { (count) } \\
3 \text { lane: } 59 \\
\text { more than } 4 \text { lanes: } 88\end{array}$ \\
\hline Ramp Type & $\begin{array}{l}\text { Ramp combination type: } \\
\text { 1. On-ramp and On-ramp } \\
\text { 2. On-ramp and Off-ramp } \\
\text { 3. Off-ramp and On-ramp } \\
\text { 4. Off-ramp and Off-ramp }\end{array}$ & $\begin{array}{l}1: 79 \text { (count) } \\
2: 21 \\
3: 71 \\
4: 35\end{array}$ \\
\hline $\begin{array}{l}\text { Average } \\
\text { Speed }\end{array}$ & $\begin{array}{l}\text { Average speed for the crashes } \\
\text { that occurred on the same } \\
\text { roadway segment }\end{array}$ & $\begin{array}{l}\text { Mean: } 44.2(\mathrm{~km} / \mathrm{h}) \\
\text { Standard Deviation: } 19.2(\mathrm{~km} / \mathrm{h})\end{array}$ \\
\hline $\begin{array}{l}\text { Traffic } \\
\text { Volume }\end{array}$ & $\begin{array}{l}\text { Average traffic volume per lane } \\
\text { for the crashes that occurred on } \\
\text { the same roadway segment }\end{array}$ & $\begin{array}{l}\text { Mean: } 121.2 \text { (pcupl per } \\
\text { roadway segment) } \\
\text { Standard Deviation: } 51.2 \text { (pcupl } \\
\text { per roadway segment) }\end{array}$ \\
\hline $\begin{array}{l}\text { Auxiliary } \\
\text { Lane Length }\end{array}$ & & $\begin{array}{l}\text { Mean: } 177.7(\mathrm{~m}) \\
\text { Standard Deviation: } 178.9(\mathrm{~m})\end{array}$ \\
\hline $\begin{array}{l}\text { Crash } \\
\text { Frequency }\end{array}$ & & $\begin{array}{l}\text { Mean: } 6.8 \\
\text { Standard Deviation: } 9.2\end{array}$ \\
\hline
\end{tabular}

Figure 1 Ramp types

Table 2 Summary statistics of the segment-based dataset 
2 For the scenario-based analysis, crashes were aggregated based on the combinations 3 of similar traffic operating conditions and geometric characteristics as employed by 4 Imprialou et al., (2016). Four key variables were used as the control variable to define the potential crash scenarios: pre-crash operating speed, traffic volume, number of lanes, and ramp types. The traffic characteristics were first grouped into categories with the help of their cumulative distributions. For instance, pre-crash speed data were classified into 25 equal groups with a 4-percentile step. Similarly, traffic volume data were divided into 4 categories with a step of 25-percentile. Finally, a total of 1,200 crash occurrence scenarios were then created (i.e. 25 speed categories $\times 4$ traffic volume categories $\times 3$ lane numbers $\times 4$ ramp types). For instance, one of the 1,200 observations is represented as speed is between the $20^{\text {th }}$ and $24^{\text {th }}$ percentile with the median value of $19 \mathrm{~km} / \mathrm{h}$, traffic volume is between $50^{\text {th }}$ and $75^{\text {th }}$ percentile with the median value of $154.6 \mathrm{veh} /$ lane on a 3-lane expressway segment with a ramp type as on-ramp and off-ramp.

Crashes were then classified into the preset 1,200 scenarios according to their traffic conditions before crash occurrence and geometric characteristics of the crash locations. Then crashes grouped into the same scenario were aggregated to formulate the analysis dataset, and the median values of speed and traffic volume within each group were utilized to represent the traffic conditions corresponding to the calculated crash frequency. Table 3 presents the summary statistics of the scenario-based dataset.

Table 3 Summary statistics of the scenario-based dataset

\begin{tabular}{|c|c|c|}
\hline Variable & Description & Summary Statistics \\
\hline Lane & Number of lanes & $\begin{array}{l}\text { \# of lanes } 2: 59 \text { (count) } \\
\text { \# of lanes } 3: 59 \\
\text { \# of lane more than } 4: 88\end{array}$ \\
\hline Ramp type & $\begin{array}{l}\text { Ramp combination type: } \\
\text { 1. On-ramp and On-ramp } \\
\text { 2. On-ramp and Off-ramp } \\
\text { 3. Off-ramp and On-ramp } \\
\text { 4. Off-ramp and Off-ramp }\end{array}$ & $\begin{array}{l}\text { Type 1: } 79 \text { (count) } \\
\text { Type 2: } 21 \\
\text { Type 3: } 71 \\
\text { Type 4: } 35\end{array}$ \\
\hline Speed & $\begin{array}{l}\text { Median speed for the preset } \\
\text { crash occurrence scenario }\end{array}$ & $\begin{array}{l}\text { Mean: } 33.6(\mathrm{~km} / \mathrm{h}) \\
\text { Standard Deviation: } 17.3(\mathrm{~km} / \mathrm{h})\end{array}$ \\
\hline $\begin{array}{l}\text { Traffic } \\
\text { Volume }\end{array}$ & $\begin{array}{l}\text { Median volume per lane for the } \\
\text { preset crash occurrence scenario }\end{array}$ & $\begin{array}{l}\text { Mean: } 127.5 \text { (pcupl per } \\
\text { roadway segment) } \\
\text { Standard Deviation: } 46.6 \text { (pcupl } \\
\text { per roadway segment) }\end{array}$ \\
\hline $\begin{array}{l}\text { Crash } \\
\text { Frequency }\end{array}$ & & $\begin{array}{l}\text { Mean: } 3.8 \\
\text { Standard Deviation: } 3.0\end{array}$ \\
\hline
\end{tabular}


It is worth mentioning that since no prior assumptions used about the functional relationships between operating speed and crash frequency for the Shanghai expressway system, different functional forms should be tested. This includes: linear, logarithmic, and quadratic. In the final analysis results, only the significant variables and the best functional forms were kept.

\section{Datasets for the crash risk analysis}

In order to conduct the individual crash level analysis, a 30-minute period traffic data prior to crash occurrence were first identified. This means that five 6-minute intervals of traffic data were obtained during the data preparation process. For example, if a crash occurred on September 13, 2013 at 8:40 p.m., traffic data from 8:10 p.m. to 8:40 p.m. (i.e. a 30-minute window) were then extracted and named as time-slices 1, 2, 3, 4, and 5, with slice 1 being the 0-6 minutes interval just before the reported crash time. Meanwhile, traffic flow characteristics (e.g. average speed, total volume, standard deviation of speed and volume, coefficient of variance for volume and speed) were calculated from 6-minute intervals. In addition, instead of only utilizing traffic related variables from the crash current segments (C), data from both upstream (U) and downstream (D) segments were incorporated. The spatial relationship between the roadway segments is shown in Figure 2. As a result, a total of 90 variables (i.e. 6 traffic flow variables $\times 3$ detector stations $\times 5$ time slices) were generated and used in the latter model estimation procedure.

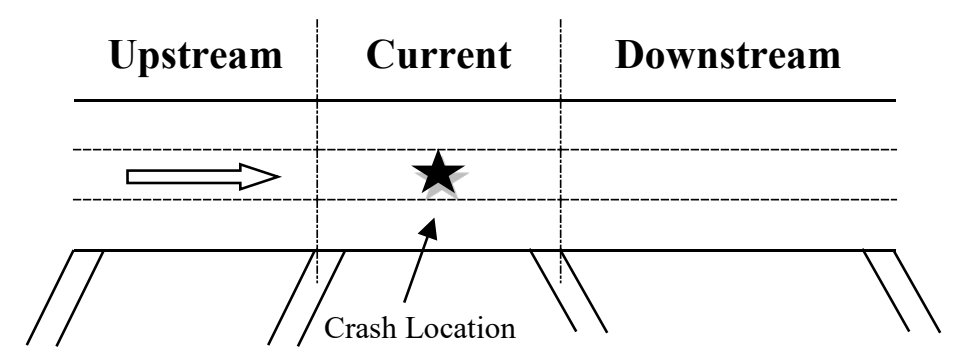

Figure 2 Arrangement of roadway sections

Since the primary idea of this part analysis is to compare normal traffic conditions with those of pre-crash conditions, traffic data from non-crash cases were also extracted. For each crash, four non-crash cases were extracted by following the matched-case control data structure as employed in existing students (e.g. Ahmed and Abdel-Aty, 2012), which was also tested in the previous sensitivity analysis. Non-crash traffic conditions were collected when no crash was observed within a 2-hour window, given the same time of day, day of week, and roadway section. For example, if a crash occurs on a segment with NN0312 (stake number) on September 13, 2013 at 8:40 p.m., traffic data for the same roadway section and time on August 31 and September 6 (i.e. two observations before the crash event) and September 20 and 
1 September 27 (i.e. two observations after the crash event) were collected as non-crash 2 cases only if there is no crash at the time period from 7:40 p.m. to 9:40 p.m. on these 3 dates. Through matching, the final dataset has 1,387 matched strata with 1,387 crashes 4 and 3,811 non-crashes (in a few cases, the non-exact 1:4 crash and non-crash ratio is 5 due to the traffic data availability issue).

\section{Methodology}

8 In order to quantify the impacts of aggregation levels on the relationships between operating speed and traffic safety, two types of models have been employed in this study: random-effects negative binomial models were used for crash frequency analyses while random-effects logistic regression models were adopted for crash risk analyses. These models were estimated by employing the Bayesian inference technique. This section introduces the model structure and the relevant inference settings.

\section{Random-effects negative binomial model}

Crash frequency data aggregated by roadway segments or by operating scenarios were assumed to follow the negative binomial distribution suitable for accounting for the over-dispersion inherent in count data (e.g. Lord and Mannering, 2010). As suggested by the previous studies (e.g. Yu et al., 2013), a random-effect term was added to account for the unobserved heterogeneity. The random effects negative binomial model can be setup as follows (Ntzoufras, 2009):

$$
\begin{gathered}
Y_{i} \sim \text { Negative Binomial }\left(p_{i}, r\right) \\
p_{i}=\mathrm{r} /\left(\mathrm{r}+\lambda_{i}\right) \\
\ln \lambda_{i}=\text { offset }_{i}+\sum_{j=1}^{k} X_{i j} \beta_{j}+u_{i} \\
\text { and } i=1,2, \ldots, n ; \quad j=1,2, \ldots, k
\end{gathered}
$$

where $Y_{i}$ is the crash count for a roadway segment $i$ or the crash count for a scenario $i$, $r$ is the dispersion parameter, $p_{i}$ and $\lambda_{i}$ are the negative binomial distribution parameters, $X_{i j}$ represent the set of explanatory variables and $\beta_{j}$ is the corresponding regression parameters to be estimated, $k$ is the number of explanatory variables and $n$ is the total number of observations. Segment length denoted as $\ln \left(\right.$ SegmentLenth $\left._{i}\right)$ can be used as the offset variable in the segment-based analysis while average vehicle-hours spent per scenario denoted as $\ln$ (AverageVehicleHours) can be used as the offset variable in the scenario-based model as suggested by Imprialou et al. (2016). $u_{i}$ is the segment/scenario specific random effect which set to follow the normal distribution with $u_{i} \sim N(0,1 / \tau)$, where $\tau$ was specified a gamma prior as $\tau \sim \operatorname{Gamma}(0.001,0.001)$. 


\section{Random-effects logistic regression model}

In the crash risk analysis, the target variable is a binary category with 1 being crash cases and 0 represents non-crash cases. Suppose observation $Y_{i}$ has the outcomes of crash and non-crash with corresponding probabilities being $\mathrm{p}_{i}$ and $1-\mathrm{p}_{i}$ respectively. The random effects logistic regression model can be set up as follows:

$$
\begin{gathered}
Y_{i} \sim \operatorname{Binomial}\left(p_{i}\right) \\
\operatorname{logit}\left(p_{i}\right)=\log \left(\frac{p_{i}}{1-p_{i}}\right)=\beta_{0}+\sum_{j=1}^{m} Z_{i j} \beta_{j}+\varepsilon_{t} \\
\text { and } i=1,2, \ldots, N ; \quad j=1,2, \ldots, m
\end{gathered}
$$

where $\beta_{0}$ is the intercept and $Z_{i j}$ is the set of explanatory variables, $\beta_{j}$ is the corresponding regression coefficients to be estimated, $m$ is the number of explanatory variables, $N$ is the number of observations $\varepsilon_{t}$ is the random effects term:

$$
\varepsilon_{t} \sim N(0,1 / \alpha)
$$

$$
\alpha \sim \operatorname{Gamma}(0.001,0.001)
$$

where $t$ stands for the crash unit index (crash observation and their matched non-crash cases). The random effects term can take into account any potential unobserved heterogeneity arising from omitted geometric characteristics not considered in the set of explanatory variables such as auxiliary lane length.

\section{Bayesian Inference}

Full Bayesian inference was employed in this study with non-informative priors. For each model, three chains of 20,000 iterations were set up in WinBUGS (Lunn et al., 2000) with the thin set equal to 3 ; the first 5,000 stored iterations were used as burn-in samples and the rest was used to estimate the poster distribution. Convergences of the developed models were checked by monitoring the MCMC (Markov chain Monte Carlo) trace plots for the parameters and the model convergence issue was further checked through calculating BGR statistics (Gelman and Rubin, 1992) and conducting the Geweke diagnostic through R package - boa (Smith, 2007).

\section{Modeling Results}

\section{Segment-based Analysis}

Table 4 shows the posterior estimations of the Bayesian random-effects negative binomial model for the segment-based dataset. Five explanatory variables became statistically significant based on their $95 \%$ posterior credible levels. For the operating speed, Av_Spd is significant with a negative coefficient, which indicates that as the operating speed increase, crash frequency would be reduced. Similar results have also been concluded in the previous study (Yu et al., 2013), which can be understood as that crashes are more prone to happen at congested segments. 
1 Besides, traffic volume - $\mathrm{Ln}(\mathrm{Vol}$ per lane) holds a positive estimate; indicating that the

2 larger traffic exposure, the larger crash frequency. For the variable representing lane

3 numbers, Lane_3 was treated as the reference group; Lane_2 shows a positive

4 association with the crash frequency whereas Lane_4 has a negative coefficient,

5 which indicates that as segments with high number of lanes are associated with lower

6 crash counts. Aux_length was found to have a significant impact on crash frequency.

7 More specifically, longer auxiliary length within the roadway segment would

8 substantially reduce crash frequency. For the ramp types, Ramp_1 was identified as

9 no substantial difference when compared to Ramp_2, while Ramp_3 and Ramp_4

10 were proved to provide lower crash hazardous.

Table 4 Coefficient estimates for segment-based analysis

\begin{tabular}{lcccc} 
Variable & Mean & S.D. & $2.5 \%$ & $97.5 \%$ \\
\hline Intercept & -3.7 & 1.36 & -6.28 & -1.12 \\
Lane_2 & 0.68 & 0.24 & 0.21 & 1.17 \\
Lane_4 & -0.46 & 0.20 & -0.86 & -0.06 \\
Lane_3 (reference) & 0 & - & - & - \\
Aux_Length & -0.002 & 0.0007 & -0.003 & -0.0007 \\
Ramp_1 & 0.067 & 0.26 & -0.45 & 0.59 \\
Ramp_2 (reference) & 0 & - & - & - \\
Ramp_3 & -1.08 & 0.31 & -1.71 & -0.46 \\
Ramp_4 & -0.48 & 0.26 & -1.02 & -0.02 \\
Av_Spd & -0.03 & 0.006 & -0.04 & -0.02 \\
Ln(Vol per lane) & 1.32 & 0.26 & 0.82 & 1.83 \\
Offset variable & 1 & \multicolumn{2}{l}{$\ln$ (Total link length) } \\
\hline Tau & 2.54 & 1.62 & 1.2 & 7.11 \\
\# of observations & \multicolumn{5}{c}{206} \\
DIC & \multicolumn{5}{c}{} \\
\hline
\end{tabular}

12

\section{Scenario-based Analysis}

Table 5 shows the estimation results for the scenario-based analysis. Both operating speed and its quadratic parameter became significant. The speed parameter holds a negative coefficient and speed quadratic parameter shows a positive impact; the relationship between operating speed and crash occurrence can therefore be regarded as a U-shaped curve. This means that crash frequency decreases as operating speed increases before a critical speed is reached. After the critical speed, crash frequency increases with the operating speed. From the estimated coefficients (see Table 4), this critical speed is predicted to be $25 \mathrm{~km} / \mathrm{h}$ for the sample data from the Shanghai Urban Expressway system. This reveals that the impact of operating speed on crashes reaches to a minimum level when the mean operating speed is about $25 \mathrm{~km} / \mathrm{h}$.

In addition, for the geometric characteristic parameters, lane numbers and ramp types were also statistically significant. Consistent results have been concluded for number 
1 of lanes with the segment-based approach, where segments with more lanes are 2 related to reduced crash occurrences. While for ramp types, Ramp_2 was identified to 3 be the most hazardous one, the combination of on-ramp and off-ramp would pose

4 large needs of traffic weavings; which is inconsistent with the segment-based analysis. 5 Furthermore, the estimation result for traffic volume (Vol per lane) is consistent with

6 the segment-based analysis, whereas the increase of volume would increase the crash 7 occurrence exposure.

Table 5 Coefficient estimates for scenario-based analysis

\begin{tabular}{lcccc}
\hline Variable & Mean & S.D. & $2.5 \%$ & $97.5 \%$ \\
\hline Intercept & 1.13 & 0.17 & 0.80 & 1.46 \\
Lane_2 & 0.61 & 0.16 & 0.28 & 0.93 \\
Lane_4 & -0.14 & 0.09 & -0.22 & -0.03 \\
Lane_3 (reference) & 0 & - & - & - \\
Ramp_1 & -0.36 & 0.11 & -0.60 & -0.16 \\
Ramp_2 (reference) & 0 & - & - & - \\
Ramp_3 & -0.37 & 0.09 & -0.55 & -0.18 \\
Ramp_4 & -0.58 & 0.18 & -0.93 & -0.22 \\
Speed & -0.025 & 0.009 & -0.045 & -0.008 \\
Speed*Speed & 0.0004 & 0.00008 & 0.0003 & 0.0006 \\
Vol per lane & 0.0038 & 0.0008 & 0.0023 & 0.0053 \\
Offset variable & 1 & \multicolumn{5}{c}{ ln(Average vehicle-hours spent } \\
Tau & 2.56 & \multicolumn{5}{c}{0.41} & 1.84 \\
\# of observations & \multicolumn{5}{c}{974} \\
DIC & \multicolumn{5}{c}{ 4252.68 } \\
\hline
\end{tabular}

Crash risk analysis model

11 In this section, disaggregate crash risk analyses were conducted to identify the 12 relationships between operating speed and individual crash occurrence probability. 13 Since it was claimed in the previous studies that crash risk analysis varies by different 14 operating conditions (Abdel-Aty et al., 2005), four speed categories were classified in 15 this study according to the operation conditions at Shanghai urban expressway system: 16 low speed (less than $20 \mathrm{~km} / \mathrm{h}$ ), medium speed (between $20 \mathrm{~km} / \mathrm{h}$ and $40 \mathrm{~km} / \mathrm{h}$ ), high 17 speed (40 km/h to $60 \mathrm{~km} / \mathrm{h}$ ), and free-flow speed (above $60 \mathrm{~km} / \mathrm{h}$ ).

19 Table 6 shows the modeling results for the crash risk analysis that considers different 20 operating speed conditions. For each model, three significant variables were achieved. 21 For low speed conditions, average speed at crash segment at time slice 1 (ASC1) 22 poses a negative relationship with crash risk, which refers to congested flow would 23 have higher crash likelihood. Traffic volume at crash segment at time slice 2 (TFC2) 24 has a positive coefficient, which indicates that the increase of traffic volume would lead to larger crash hazardous. In addition, speed standard deviation of downstream 
1 segment time slice 1 (SSD1) has a positive coefficient, which can be understood as

2 larger speed variation at downstream would enhance the crash risk.

4 While for moderate speed conditions, ASC1 again holds a negative coefficient and the 5 speed standard deviation of crash segment at time slice 1 (SSC1) has a positive 6 coefficient, which can be illustrated as smoother and more homogenous traffic would 7 lead to reduced crash probability. Besides, upstream traffic volume standard deviation 8 at time slice 1 (SFU1) has a positive coefficient, which means that the variation of 9 upstream flow would enhance the crash occurrence likelihood.

11 In addition, an interesting finding is that instead of $\mathrm{ASC} 1$, the average speed at 12 downstream segment time slice 1 (ASD1) was found to provide more substantial 13 impacts on crash occurrence likelihood, while average operating speed at crash 14 locations does not have substantial correlations with crash occurrence. Furthermore, 15 standard deviation of traffic volume at crash segment time slice 1 (SFC1) and SSC1 16 both have positive coefficients, which means turbulence traffic would lead to larger 17 crash hazardous.

19 Furthermore, for the free-flow conditions, SSC1 and SFC1 hold consistently 20 estimated coefficients. However, the ASC1 has a positive sign, which indicates that as 21 the increase of operating speed, the crash risk would be also increased. This is a 22 contradictory of the results identified in the low speed condition and moderate speed 23 condition. 
Table 6 Coefficient estimates for crash risk analysis by speed conditions

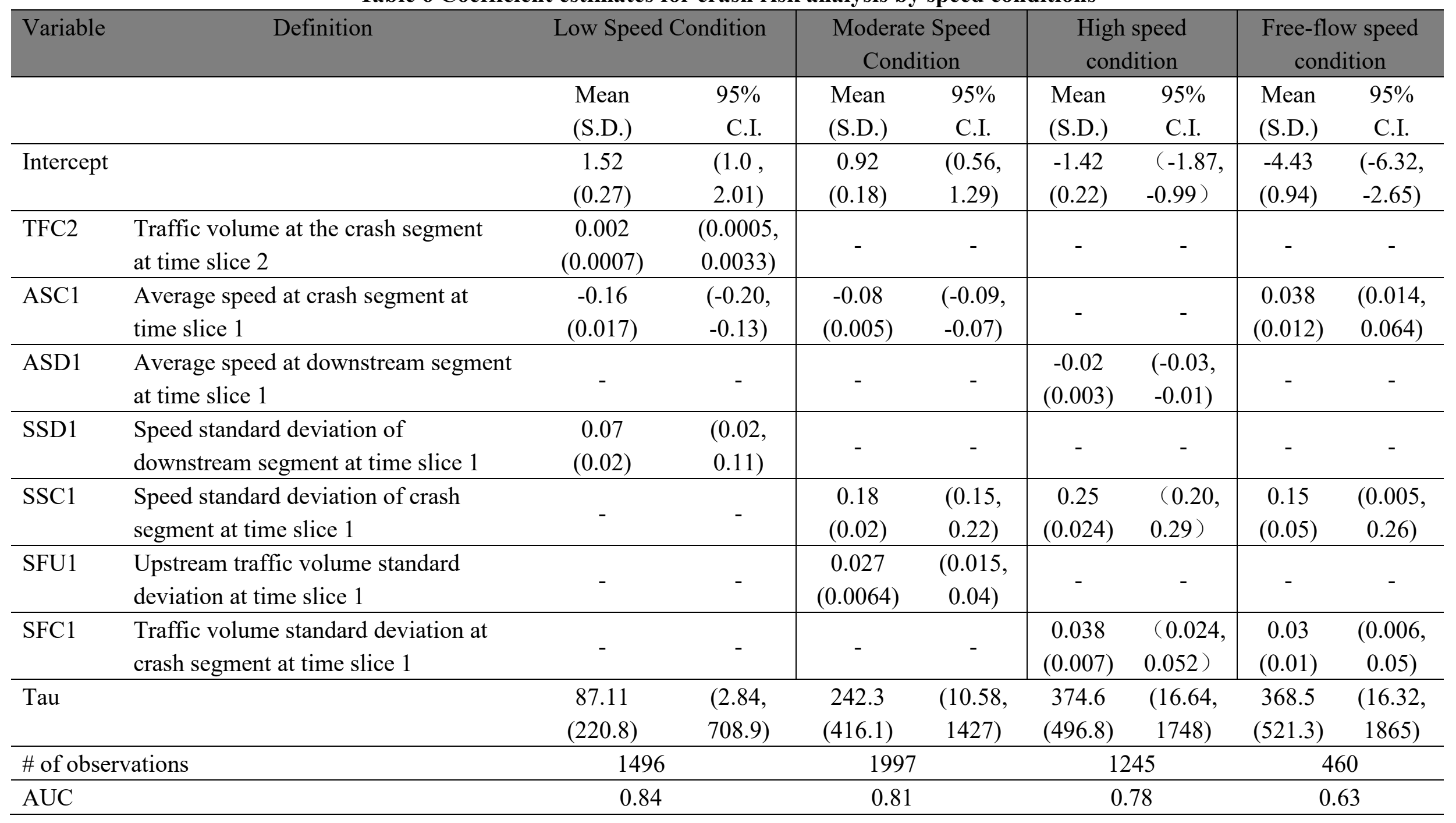


1 Therefore, the relationships between operating speed and traffic safety at crash

2 individual aggregation level is concluded as: operating speed has negative impacts on

3 crash occurrence risk under low and moderate speed conditions, at high speed

4 conditions the impacts of speed on crash occurrence is vague, while at free-flow conditions speed holds positive impacts. The modeling results indicate that the relationship between operating speed and traffic safety do not hold a linear line, it varies at different operation conditions.

\section{$8 \quad$ Discussions and Conclusions}

9 Emerging active safety management systems, such as Variable Speed Limits System or in-vehicle speed advisory system under Connected Vehicle (CV) scenario, require deep understandings of the relationships between operating speed and crash occurrence. As alluded earlier that most previous studies however used spatio-temporal average speed instead of speed information prior to crash occurrence in their analyses due to the data aggregation issue. As a result, there were no consistent findings being obtained as the over-aggregated data might fail to reveal the true association between the two.

In this study, the impacts of aggregation approaches on the relationship analyses were investigated based on the advanced traffic sensing data of Shanghai urban expressway systems. Crash frequency analyses with segment-based approach and scenario-based approach were firstly being conducted, and then crash risk analyses were developed at individual crash level. The segment-based crash frequency analysis revealed a negative relationship between the two. On the other hand, as shown in Figure 3, the results from the scenario-based crash frequency analysis, average crashes per kilometer are relatively high at both low speed traffic conditions and high speed conditions; the relationships between operating speed and crash occurrence were therefore concluded as a U-shape curve.

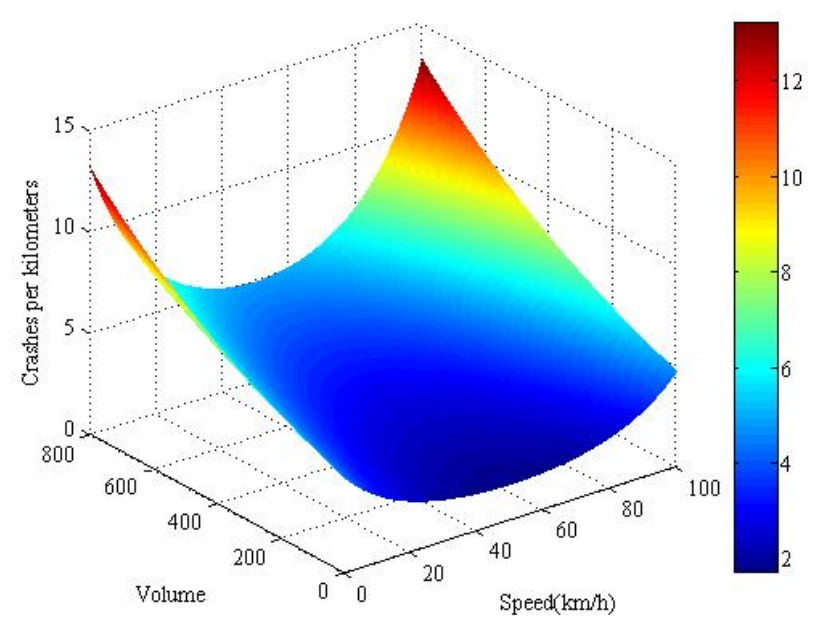

Figure 3 Relationships between speed, volume and crash rates from scenario-based crash frequency analysis 
2 Given the inconsistent results obtained from the crash frequency analyses, 3 disaggregate crash risk analyses were further conducted. Figure 4 shows the box plot 4 of the estimated coefficients for the operating speed parameter (ASC1) and Table 7 5 shows the estimated marginal effects of parameter ASC1, where the coefficient of 6 ASCI indicates the crash occurrence likelihood and operating speed. It can be 7 concluded that during the congestion period (i.e. low and moderate speed conditions), 8 the increase of operating speed would reduce a crash likelihood; for medium 9 operating speed the changes of operating speed do not have substantial effects on 10 crash occurrence probability; while for free-flow, the increase of operating speed 11 would further enhance crash hazardous.

13 The crash risk analyses have been an important topic in the traffic safety analysis 14 discipline in which different study area and research objectives have been investigated. 15 The earlier studies were mostly conducted based on total crashes and have identified 16 that the coefficient of variation of speed was the crash occurrence contributing factor 17 (Lee et al., 2003, Abdel-Aty et al., 2004), which could be understood as lower 18 operating speed and large speed variation would lead to more crashes. Recently, a few 19 studies investigated the effect of different operating conditions on safety. For instance, 20 Pande and Abdel-Aty (2006) investigated the rear-end crash occurrence influencing 21 factors, and the crashes were separated into low speed and high speed conditions. Their 22 findings are consistent with this current study where speed is positively associated with 23 traffic crashes for high operating speed conditions; while in the low speed conditions, 24 larger coefficient of variation of speed would lead to increased crash risk. However, 25 instead of split crashes by operating conditions, majority crash risk analyses divide 26 crashes by crash types (Christoforou et al., 2011), weather conditions (Xu et al., 2013), 27 and crash injury severity (Yu and Abdel-Aty 2014). But inconsistent findings reappear 28 which may be due to the heterogeneity effect resulting from different operating 29 conditions. For instance, Oh and Kim (2010) identified a positive correlation between 30 speed and crash for rear-end crashes while Christoforou et al., (2011) found a negative 31 association. Therefore, based on the current findings, it is advisable that further crash 32 risk analyses shall consider the heterogeneity effects of operating speed on traffic 33 safety. 


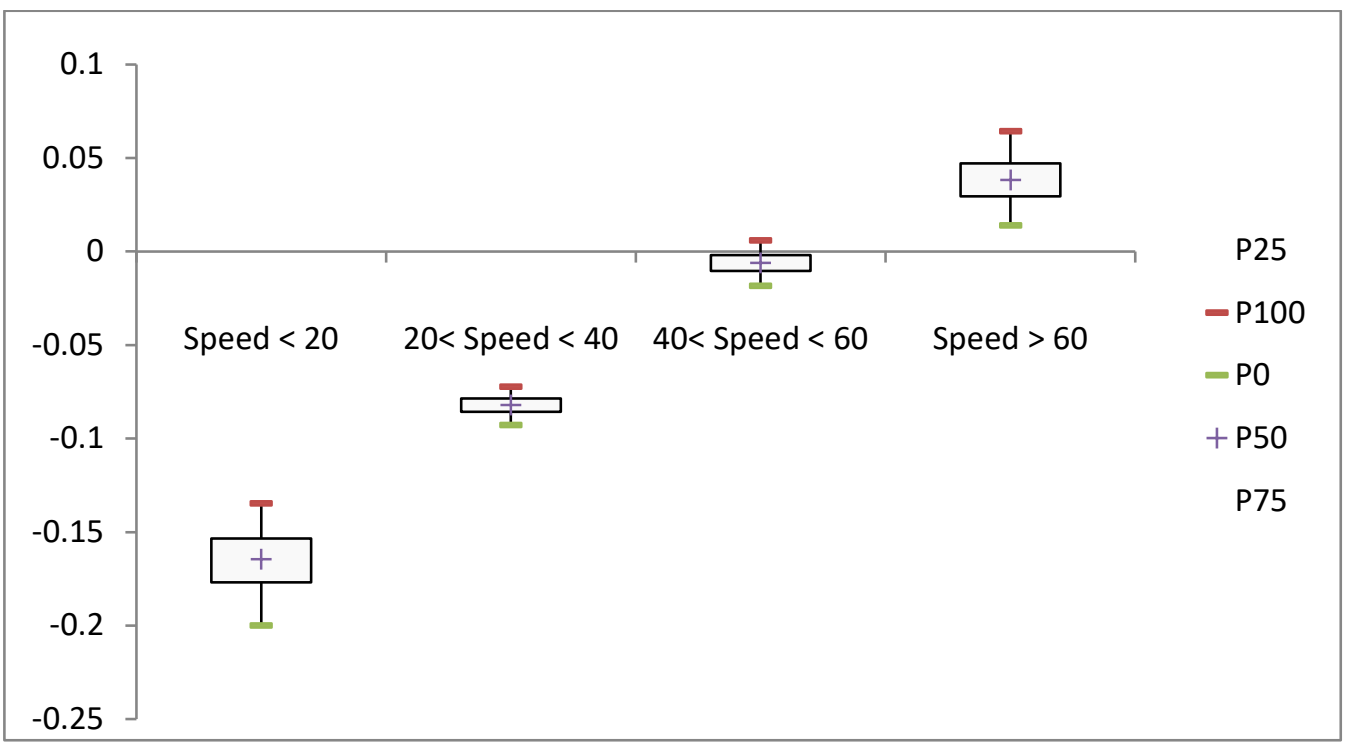

Figure 4 Box-plot for estimated coefficients of ASC1 in the crash risk analyses

Table 7 Coefficient marginal effects for ASC1

\begin{tabular}{|c|c|}
\hline Speed Conditions & Marginal Effects for ASC1 \\
\hline Low Speed Condition & -0.01649 \\
\hline Moderate Speed Condition & -0.00345 \\
\hline High Speed Condition & $-0.000521^{*}$ \\
\hline Free-flow Speed Condition & 0.00399 \\
\hline
\end{tabular}

\footnotetext{
* Insignificant marginal effect at 95\% level
}

Through comparisons, results of the crash risk analyses are consistent with the scenario-based approach crash frequency analysis. A U-shape curve relationship may be a better illustration between the operating speed and traffic safety. The linear relationship exits in the segment-based approach may be attributed to the data aggregation process; during the aggregation, crashes with high speed would be averaged by medium or low speed crash-prone speed, which leads to a monotonous relationship between speed and safety. Therefore, the scenario-based aggregation approach and crash risk analysis by speed categories are more plausible and preferred for future studies with similar objectives.

In addition, through the crash risk analyses, typical crash occurrence scenarios can be speculated with the significant contributing factors. For low speed conditions, crashes are mostly likely to happen within congested segments, where traffic flow dissipates at its downstream segment. At moderate speed conditions, crashes occurred at turbulence flow segment while its upstream has a large traffic flow. While at high speed conditions, crashes are more likely to occur at the end of shockwave propagation segment where its downstream segments were congested. In addition, for crashes occurred under free-flow conditions, the crash causations are mostly related to the unexpected traffic turbulence. With these profound understandings of crash 
1 mechanisms, targeted ATMS could be designed to improve traffic safety for the urban

2 expressway system.

3

4 Moreover, findings from this study should be carefully interpreted as the detailed 5 design data were not obtained for the studied area, and some roadway geometry 6 variables (e.g. degree of curvature, gradient) were not included. Additionally, it would 7 also be interesting to analyze the impacts of statistical modeling approach on the 8 relationships. For instance, applying models such as random-parameter negative 9 binomial model, finite-mixture models rather than a random-effect negative binomial 10 model employed in this study. Last but not the least, another important factor that 11 needs an attention is the impact of speed variation (Pei et al., 2012).

\section{Acknowledgement}

13 This study was jointly sponsored by the Chinese National Natural Science Foundation 14 (NSFC 71771174 and 71531011) and the 111 Project (B17032). 


\section{Reference}

Aarts, L., Schagen, I., 2006. Driving speed and the risk of road crashes: A review. Accident Analysis and Prevention, Vol. 38, pp. 215-224.

Abdel-Aty, M., and Radwan, E., 2000. Modeling traffic accident occurrence and involvement. Accident Analysis \& Prevention, Vol. 32, No. 5, pp. 633-642.

Abdel-Aty, M., Abdalla, F., 2004. Linking roadway geometrics and real-time traffic characteristics to model daytime freeway crashes: Generalized estimating equations for correlated data. Transportation Research Record: Journal of the Transportation Research Board, 1897(1), 106-115.

Abdel-Aty, M., Uddin. N., Pande, A., 2005. Split models for predicting multivehicle crashes during high-speed and low-speed operating conditions on freeways. Transportation research record: journal of the transportation research board, No. 1908, pp. 51-58.

Ahmed, M, and Abdel-Aty, M., 2012. The Viability of Using Automatic Vehicle Identification Data for Real-Time Crash Prediction. IEEE Transactions on Intelligent Transportation Systems, Vol. 13, No. 2, pp. 459-468.

American Association of State Highway and Transportation Officials (AASHTO), 2010. Highway Safety Manual.

Christoforou, Z., Cohen, S., Karlaftis, M., 2011. Identifying crash type propensity using real-time traffic data on freeways. Journal of Safety Research, 42(1), 43-50.

Elvik, R., 2013. A re-parameterisation of the Power Model of the relationship between the speed of traffic and the number of accidents and accident victims. Accident Analysis \& Prevention, Vol. 50, pp. 854-860.

Elvik, R., Vaa, T., 2004. The Handbook of Road Safety Measures. Elsevier, Oxford.

Fitzpatrick, C., McKinnon. I., Tainter. F., Knodler, M., 2016. The application of continuous speed data for setting rational speed limits and improving roadway safety. Safety science, Vol. 85, pp. 171-178.

Gelman, A., Rubin, D., 1992. Inference from Iterative Simulation Using Multiple Sequences. Statistical Science, Vol. 7, pp. 457-511.

Imprialou, M., Quddus, M., Pitfield, D., Lord, D., 2016. Re-visiting crash-speed relationships: A new perspective in crash modelling. Accident Analysis \& Prevention, Vol. 86, pp. 173-185.

Kloeden, C, McLean, A., Glonek, G., 2002. Reanalysis of travelling speed and the rate of crash involvement in Adelaide South Australia. Report No. CR 207. Australian Transport Safety Bureau ATSB, Civic Square, ACT

Lee, C., Hellinga, B., Saccomanno, F., 2003. Real-time crash prediction model for application to crash prevention in freeway traffic. Transportation Research Record: Journal of the Transportation Research Board, 1840 (1), 67-77.

Lord, D., Mannering, F., 2010. The statistical analysis of crash-frequency data: A review and assessment of methodological alternatives. Transportation Research Part A, Vol.44, pp. 291-305.

Lunn, D., Thomas, A., Best, N., Spiegelhalter, D., 2000. WinBUGS-a Bayesian 
modelling framework: concepts, structure, and extensibility. Statistics and Computing, Vol.10, No.4, pp.325-337.

Mirshahi, M., Obenberger, J., Fuhs, C., Howard, C., Krammes, R., Kuhn, B., Mayhew, R., Moore, M., Sahebjam, K., Stone, C., 2007. Active traffic management: the next step in congestion management.

Moreno, A., García, A., 2013. Use of speed profile as surrogate measure: Effect of traffic calming devices on crosstown road safety performance. Accident Analysis \& Prevention, Vol. 61, pp. 23-32.

Ntzoufras, I., 2009. Bayesian modeling using WinBUGS. WILEY.

Pande, A., Abdel-Aty, M., 2006. Comprehensive analysis of the relationship between real-time traffic surveillance data and rear-end crashes on freeways. Transportation Research Record: Journal of the Transportation Research Board, 1953 (1), 31-40.

Pei X, Wong, S., Szeb, N., 2012. The roles of exposure and speed in road safety analysis. Accident Analysis \& Prevention, Vol. 48, pp. 464-471.

Quimby, A., Maycock, G., Palmer, C., Buttress, S., 1999. The factors that influence a driver's choice of speed: a questionnaire study. TRL Report No. 325. Transport Research Laboratory TRL, Crowthorne, Berkshire.

Roshandel S., Zheng Z., Washington S., 2015. Impact of real-time traffic characteristics on freeway crash occurrence: Systematic review and meta-analysis. Accident Analysis \& Prevention, Vol.79, pp. 198-211.

Smith, B., 2007. Boa: An r package for MCMC output convergence assessment and posterior inference. Journal of Statistical Software, Vol.21, pp.1-37.

Taylor, M., Lynam, D., Baruya, A., 2000. The effects of drivers' speed on the frequency of road accidents. TRL REPORT 421.

Xu, C., Tarko, A., Wang, W., Liu, P., 2013. Predicting crash likelihood and severity on freeways with real-time loop detector data. Accident Analysis \& Prevention, 57, 30-39.

Yu, R., Abdel-Aty, M., Ahmed, M., 2013. Bayesian random effect models incorporating real-time weather and traffic data to investigate mountainous freeway hazardous factors. Accident Analysis \& Prevention, Vol. 50, pp.371-376.

Yu, R., Abdel-Aty, M., 2014. Analyzing crash injury severity for a mountainous freeway incorporating real-time traffic and weather data. Safety Science, 63, $50-56$. 